\title{
Histological and Clinical Findings in Rabbits Sensitized with GM1 Ganglioside
}

\author{
Ni Komang Sri Dewi Untari ${ }^{1,2 *}$, Kurnia Kusumastuti ${ }^{3}$, Guritno Suryokusumo ${ }^{4}$, I. Ketut Sudiana ${ }^{5}$, Tedy Juliandhy ${ }^{6}$ \\ ${ }^{1}$ Department of Hyperbaric, Drs. Med. Rijadi S. Phys. Naval Health Institute, Surabaya, Indonesia; ${ }^{2}$ Doctoral Program, Faculty \\ of Medicine, Airlangga University, Surabaya, Indonesia; ${ }^{3}$ Department of Neurology, Faculty of Medicine, Airlangga University, \\ Surabaya, Indonesia; ${ }^{4}$ Department of Hyperbaric, Faculty of Medicine, Pembangunan Nasional University, Jakarta, Indonesia; \\ ${ }^{5}$ Department of Pathology Anatomy, Faculty of Medicine, Airlangga University, Surabaya, Indonesia; ${ }^{6}$ Department of Electrical \\ Engineering, Hang Tuah University, Surabaya, Indonesia
}

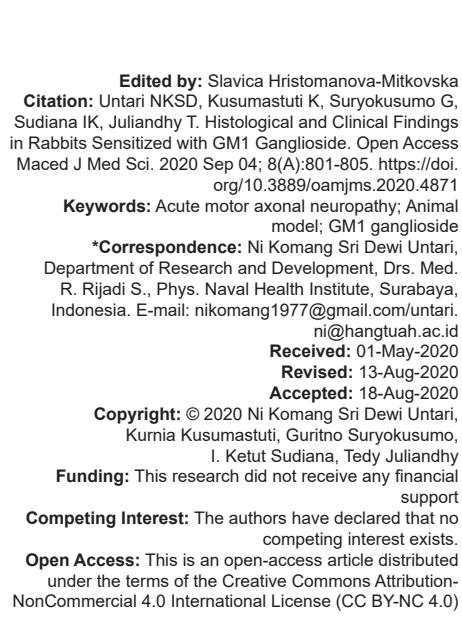

\section{Abstract}

BACKGROUND: Acute motor axonal neuropathy (AMAN) is a peripheral nerve disorder that attacks motor axons and occurs acutely. AMAN is one type of Guillain-Barre syndrome (GBS) which often attacks men of productive age. Until now, although patients have undergone intravenous immunoglobulin (IVIG) therapy and/or plasmapheresis, long-standing disability remains a problem. In Indonesia, the availability and cost of these therapies are constraints.

AIM: Our study aimed to find a proper animal model suitable for AMAN and can be executed in our institution, Naval Health Institute with a hope to find new therapeutic modalities in healing with AMAN.

METHODS: GM1 ganglioside immunized in New Zealand male white rabbits with complete Freund's adjuvant, every 3 weeks until 20 weeks. We evaluated the effects GM1 ganglioside on body weight, functional score, and axon degeneration's scale. Functional score was examined based on Tarlov's. Hematoxylin-eosin was used to stain this slide.

RESULTS: Rabbits that being immunized with GM1 ganglioside experience a number of neurological signs and symptoms that resemble AMAN, that is, sluggish righting reflex, muscular weakness, flaccid hyper paralysis, and body weight loss. Pathological examination shows extensive degeneration of peripheral nerves, infiltration of macrophages, and perineuritis.

CONCLUSION: This histological and clinical findings support that this neuropathy is induced by an autoimmune response delivered by cells that respond to gangliosides.

\section{Introduction}

Acute motor axonal neuropathy (AMAN) is a primary axonal disorder that affects only the motor nerves and the second most type of Guillain-Barre syndrome (GBS). GBS is an autoimmune disease with demyelinating peripheral nerves that cause ascending and progressive muscle weakness [1]. AMAN is $10-20 \%$ of GBS sporadic cases that have an incidence of about $1 / 100,000$ [2]. The incidence increases from $0.62 / 100.00$ children to $2.66 / 100,000$ ages $80-89$ years. The ratio of male to female is $3: 2$. Neurological symptoms last up to $20 \%$ with severe disabilities. More than $80 \%$ occur severe fatigue and persist after resolution of other symptoms [3]. Mortality rates in Europe and North America vary between 3 and 7\% [4]. Intravenous immunoglobulin (IVIG) and plasmapheresis (PE) are effective for GBS patients if given the first few weeks. Even though, persistent disability is seen in $20-30 \%$ of adult GBS patients [5]. In Indonesia, however, both modalities are quite expensive. For this reason, the researcher decided to try finding other alternative therapeutic options using animal models that could represent AMAN.

There have been three ways to model animals for neurological dysfunction in GM1-immunized animals. The first way is by Nagai, GM1-immunized rabbits experience spastic paralysis. Histologically, phagocytic cells containing myelin debris can be observed. Another study by Thomas, rabbits experienced subclinical neuropathy. Mild axonal degeneration of the sciatic nerve and IgM deposits in Ranvi nodes is obtained. This discovery did not occur in mice. Conversely, sensitization of rabbits with GD1b induces sensory neuropathy associated with anti-GD1b antibodies. This confirms that failure to induce neuropathy by sensitizing with ganglioside depends on the susceptibility of the species and to the immunization procedure used [6]. Therefore, in the third study, rabbits were inoculated with a mixture of bovine brain ganglioside (BBG) according to the Kusunoki et al. procedure. Rabbits have AMAN associated with anti-GM1 IgG antibodies. Experimental motor axonal neuropathy is also induced by sensitization with GM1 [7]. 
The purpose of this journal is to explain the results obtained in making an AMAN animal model according to Yuki in our hyperbaric laboratory.

\section{Materials and Methods}

The researcher modeled the axonal GBS type by imitating the modified Yuki method. Male New Zealand white rabbit, weighing $2.0 \mathrm{~kg}$, obtained from Tuban Animal Husbandry. These rabbits were given 0.5 mg ganglioside GM1 dissolved in $0.5 \mathrm{ml}$ limpet keyhole hemocyanin $(\mathrm{KLH})$ in phosphate buffer saline (PBS). Half an mg of the complete Freund supplement (FCA) is added and the mixture emulsified. A $1 \mathrm{ml}$ GM1 emulsion sample was injected subcutaneously on the back and intraperitoneally at intervals of 3 weeks until there was a weakness in the locomotion. Rabbits are checked daily for clinical signs and weighed once a week. All animal procedures refer to the institutional guidelines of the Faculty of Veterinary Medicine, Airlangga University.

Motor strength according to Tarlov modification [8] is no limb function - back or dead (0), only joint motion (1), active motion but unable to stand (2), able to stand but unable to walk (3), and normal movement function (4).

Variable measurement studies were conducted in 14 rabbits, divided into two groups. The first seven groups were normal rabbits and the last were rabbits that inoculated with GM1. All data were analyzed using IBM SPSS version 22.0. The significance level for statistical analyzes $p<0.05$ was considered statistically significant.

\section{Pathological studies}

Rabbits were deeply anesthetized by an intraperitoneal injection of ketamine. The right sciatic nerves and the lumbar spine were removed for light microscopic analysis. Nerve specimens were immersed directly in $10 \%$ formaldehyde and stained with hematoxylin-eosin and examined. The preparations were blindly assessed by pathology anatomist, who was blinded to the experimental procedure and sampled region. Differences in the frequency distribution of macrophage infiltration and perineuritis were statistically examined with the Mann-Whitney U-test.

The image analysis program Mascope was used for morphological examination of the sciatic nerves. Digital photo images were obtained with a light microscope with a final magnification of $\times 400$ and an attached CCD camera interfaced with a computer. Both the central and peripheral nervous system are assessed. For the central nervous system, in this case, the lumbar vertebrae, the number of macrophages, and neuron edema are assessed. The number of macrophages is calculated per three visual fields and the average is taken. Neuronal edema is measured manually by the distance between the walls of the neuron and its neurons in both the crosssection and the mean were taken. As for the peripheral nervous system, what is concerned is the pattern of undulation. This undulation pattern is measured manually by drawing a straight line between two formations such as a mountain as far as $2 \mathrm{~cm}$ and reading the height of a straight line. The scoring of amount of neuron was undertaken width of neuron edema, neutrophil infiltration, and perineuritis on a scale of 1-4 as follows: $1=$ none or minor; 2 = modest; 3 = intermediate; and 4 = prominent.

\section{Results}

\section{function}

Effects of GM1 in body weight and motor

Of the 10 control rabbits, none show weakness locomotor until the end of examination time (20 weeks). In contrast, all 10 rabbits immunized with IgGM1 show flaccid paresis of locomotor hind, with the start time of events ranging from 71 to 107 days (mean 92 days) after the first inoculation. Seven rabbits immunized GM1, begin to experience weight loss in $<8$ weeks. Weight reduction is in accordance with the conditions of rabbits began inexhaustible food rations and drinking. When these rabbits start weight loss consistent for 2 weeks, it is considered as the onset of the disease. After experiencing weight loss, they start to have weakness of locomotor. The weight on this group was calculated when the terminated decline of about $0.1-0.3$ is calculated from the initial onset of clinical sign (week 8-10) (Table 1).

Table 1: Rabbits inoculated with GM1

\begin{tabular}{|c|c|c|c|c|c|c|c|c|c|}
\hline Rabbit & $\begin{array}{l}\text { Inoculation } \\
\text { times }\end{array}$ & $\begin{array}{l}\text { The onset of limb } \\
\text { weakness }\end{array}$ & $\begin{array}{l}\text { Days from onset } \\
\text { to peak }\end{array}$ & $\begin{array}{l}\text { Poorest functional } \\
\text { grade }\end{array}$ & $\begin{array}{l}\text { Body weight } \\
\text { at } 0^{\text {WK }}\end{array}$ & $\begin{array}{l}\text { Body weight at } \\
10^{\text {wK }}\end{array}$ & $\begin{array}{l}\text { Bodyweight at } \\
16^{\text {WK }}\end{array}$ & $\begin{array}{l}\text { Body weight } \\
\text { at } 20^{\text {WK }}\end{array}$ & $\begin{array}{l}\text { Axon degenerative } \\
\text { scale }\end{array}$ \\
\hline GM1-1 & 4 & 97 & 6 & 2 & 2,1 & 2.4 & 2.3 & 2.3 & 4 \\
\hline GM1-2 & 4 & 71 & 26 & 0 & 2.2 & 2.4 & 2 & 2.0 & 3 \\
\hline GM1-3 & 4 & 94 & 29 & 3 & 2.1 & 2.5 & 2.2 & 2.2 & 4 \\
\hline GM1-4 & 4 & 75 & 33 & 0 & 2.0 & 2.3 & 2.1 & 2.1 & 4 \\
\hline GM1-5 & 4 & 99 & 38 & 1 & 1.8 & 2.2 & 2 & 2.0 & 4 \\
\hline GM1-6 & 4 & 103 & 17 & 2 & 2.2 & 2.1 & 2.2 & 2.2 & 4 \\
\hline GM1-7 & 4 & 107 & 23 & 2 & 2.0 & 2.2 & 2.3 & 2.3 & 3 \\
\hline $\mathrm{N}-1$ & 0 & 0 & 0 & 4 & 2.2 & 2.5 & 2.7 & 2.9 & 1 \\
\hline $\mathrm{N}-2$ & 0 & 0 & 0 & 4 & 2.2 & 2.4 & 2.8 & 3.0 & 1 \\
\hline $\mathrm{N}-3$ & 0 & 0 & 0 & 4 & 2.1 & 2.5 & 2.6 & 2.9 & 1 \\
\hline $\mathrm{N}-4$ & 0 & 0 & 0 & 4 & 2 & 2.5 & 2.7 & 2.8 & 1 \\
\hline $\mathrm{N}-5$ & 0 & 0 & 0 & 4 & 2 & 2.5 & 2.6 & 2.7 & 1 \\
\hline $\mathrm{N}-6$ & 0 & 0 & 0 & 4 & 1.8 & 2.4 & 2.7 & 2.8 & 1 \\
\hline $\mathrm{N}-7$ & 0 & 0 & 0 & 4 & 1.9 & 2.5 & 2.6 & 2.9 & 1 \\
\hline
\end{tabular}


Rabbits GM1-3, GM1-4, and GM1-7 had tremors a few days before the start of locomotor weakness. Weakness locomotor becomes progressively during 4-13 days (median 5 days) after the start of the weakness in seven rabbits until it reaches a constant value. Tetraparesis occurs rapidly in rabbits GM1-2 and GM1-4, with breathing became labored and gasping. After the commencement of locomotor weakness, they die, respectively, on day 36 and 41 (Figures 1 and 2).

At approximately the same time, the equivalent of rabbits in the control group terminated. There is no weight loss in the control group. Instead, the weight raised up ranged 0.7 until $1 \mathrm{~kg}$. There were significantly different between body weights from two groups at week $16(p=0.002)$ and $20(p=0.002)$.
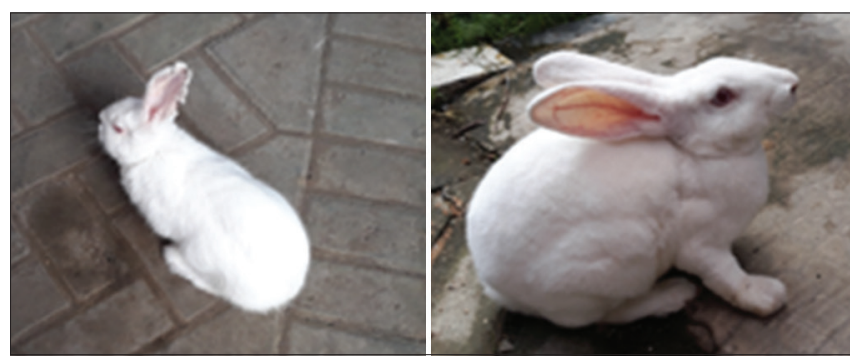

Figure 1: Rabbit GM1-1 (left) at the beginning of the study and rabbit Cr-1 (right) at the end of the study. Rabbits with straight ears can maintain a normal standing position and lift the head and body.

\section{Histological findings}

There were no changes in the bone marrow or the sciatic nerves of all rabbits that were not immunized GM1. In lumbar fragments painted with $\mathrm{HE}$, tissue patterns can be observed, which make it possible to recognize gray matter and white matter, even though

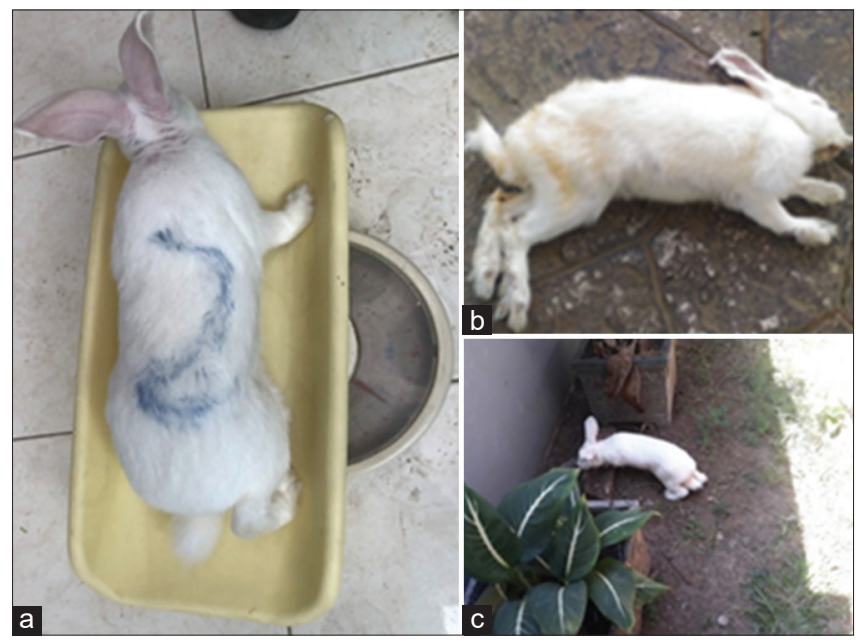

Figure 2: (a) GM1-2 rabbit with locomotion weakness 10 days after onset. Rabbit cannot maintain a normal standing position or lift their head and body. Muscle locomotion and body are weak and sagging, giving less resistance generally in passive movements. (b) GM1-5 rabbit with locomotion weakness 15 days after onset. The rabbit is less capable of moving the hint, moving only with both hands. They try to jump but cannot. (c) GM1-4 rabbit with locomotion weakness 29 days after onset. Forty-one days after onset, the rabbit dies, there is no vital sign. the boundary between the two areas is similar and difficult to identify. Whereas in the analysis of normal sciatic nerves with HE staining, it is possible to observe tissue patterns with special undulation and parallel organization of nerve fibers. Myelin sheath painting is weak and unspecified, with very low contrast between various histological structures.

The sagittal (left) sacral section of a normal rabbit found less than 20 macrophages infiltration. The neuron cells size appear normal, without interneuron edema. The longitudinal (right) section shows a unique undulation pattern with a straight line-height between two mountain-like formations over $3 \mathrm{~cm}$. There were no signs of axon degeneration in the normal ischiadica nerve's rabbits.

Morphological analysis by HE painting after 20 weeks of in vivo immunization enables observing axonal degeneration. The number of macrophages increases with minimal lymphocyte infiltration. Interneuron edema increases approximately $1-2.4 \mathrm{~mm}$ at $\times 400$. There are also neurons with apoptotic material. There was no difference in the number of blood vessels in the two groups.

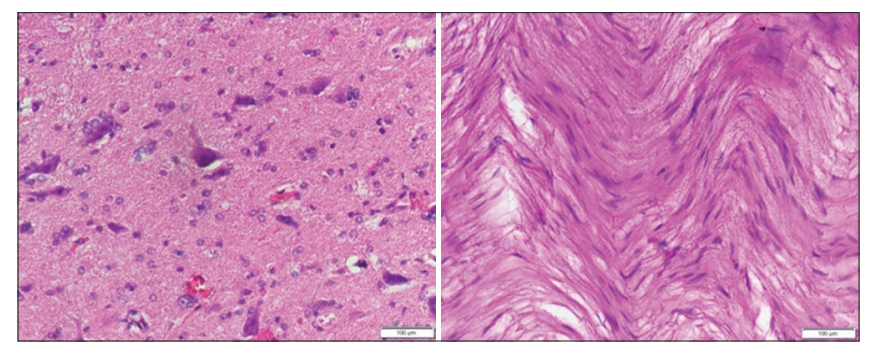

Figure 3: The sagittal (left) section of the normal rabbit myelum is seen with normal macrophage infiltration and neuron cells with normal size, without intraneuronal edema. Longitudinal (right) chunks of the normal rabbit are chiadika nerve appear typical undulation patterns.

In the sciatic nerve, a typical undulation tissue pattern is observed from the peripheral nerve with Wallerian degeneration. Histochemical reactions to collagen fibers are intense and fibers tend to stay oriented in place. In longitudinal sections, structural disorganization with a straight line height between the two mountain-like formations is $<2.5 \mathrm{~cm}$. In some rabbits, this undulation is almost completely gone. There were also significantly different between axon degenerations at week $20(p=0.02)$ (Figures 3 and 4).

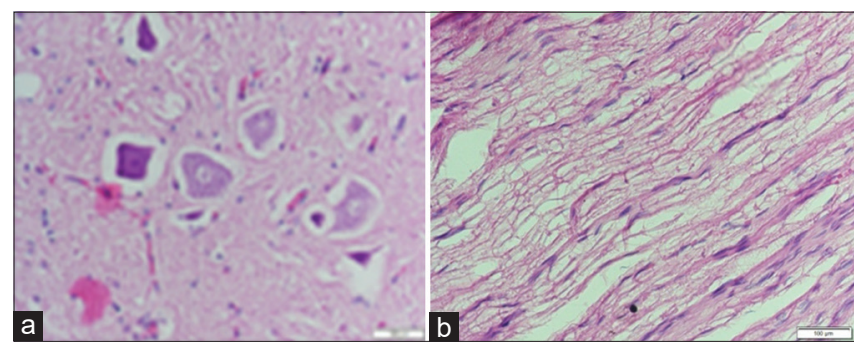

Figure 4: (a) Sagittal cross-section of the sacral (left) shows macrophage infiltration and neuronal edema in rabbit-immunized $\mathrm{HE}$ prepared rabbits. (b) Longitudinal cross-section of the sciatic nerve (right) showing a flat pattern of undulation. 


\section{Discussion}

GBS is an acute disease that attacks the peripheral nerves. The disorder commonly presents with a weakness that is often symmetric and most often begins in the legs. Autonomic involvement is common and may lead to a fatal outcome, as may aspiration pneumonia or impaired respiration from weakness [9]. GBS has a serious long-term impact on the patients' work and private life, even 3-6 years after the onset of the illness [5].

Several subtypes of GBS are now recognized, as determined primarily by electrodiagnostic and pathologic distinction. Although they have received standard therapy, namely, IVIG and plasmapheresis, the disability remains a problem [9]. Axonal type is the most common type in Indonesia with AMAN being the most frequent. The cost of standard therapy is a problem. In our institution, AMAN patients who receive oxygen hyperbaric get a faster motor repair. We try to reduce it to research using experimental animals. Unfortunately, AMAN model does not yet exist in Indonesia.

The use of the rabbit as an animal model for studying human biology is based on the phylogenetic relationship, the similarity of anatomy, physiology, and behavior of humans. Rabbits have an advantage with the size of the middle between rodents and larger animals and more expensive, such as primates. Rabbit size allows blood samples sap and greater access to many cells and tissues. In addition, rabbits have a life span longer than rodents, and genes of the immune system of rabbits appear to be more similar to the human immune system genes than rodents. Rabbits also are carriers or reservoirs of some zoonotic pathogens that cause disease.

Of the seven rabbits given GM1 immunization, we got signs and weakness symptoms that were very similar to patients with GBS axonal type. Nagai observed that rabbits immunized intensively with bovine brain ganglioside or certain molecules from ganglioside species, such as GD1a and GM1 in CFA will experience various neurological symptoms and signs that closely resemble experimental allergic encephalomyelitis [10]. Some rabbits experience initial symptoms such as tremors. On average at week 8 , rabbits begin to experience a decrease in appetite and drinking. Two weeks after the decrease in intake, weight loss was recorded from 0.1 to $0.2 \mathrm{~kg}$. Clinical symptoms in the form of motor weakness appear a week after this with a decrease in the agility of the back motion until it can only move the front. Two rabbits die on the day 36 and 41 after symptoms begin.

In terms of pathology observations, painting hematoxylin and eosin ( $\mathrm{H} \& \mathrm{E})$ is the easiest and the most frequent staining that is often used for light microscopy and even for peripheral nerve [11]. It is known that light microscopy can detect peripheral nerve damage. Even though this strain is not an ideal method for analyzing the nerve regeneration process, its electropolar nature can make H\&E used. As we know, this stain will produce pink and purple color with various intensities in neural elements. Any degenerative nerve will be shown as vacuole (vacuolated foci). An empty zone is the characteristic of myelin with this staining [12].

The sciatic nerves showed severe to mild Wallerian-like degeneration, with an increasingly flat form of undulation. There is the invasion of macrophage in the endoneurial-perivascular region, but there was not any infiltration of lymphocytes in any of sciatic nerve regions. These results suggest that this syndrome may be induced by cell-delivered autoimmune responses that respond to gangliosides [7]. Thomas stated mild axon degeneration and immunoglobulin deposits in the Ranvier node in the peripheral nerves, resembling morphic neuropathy patients, motor conduction blocks, and anti-GM1 antibodies [6].

At GBS cases, molecular mimicry happens. Microbial antigens may cross-react with self-antigens. Therefore, immune responses initiated by the microbes may activate $T$ cells specific for self-antigens [13]. In the autopsy case, perivascular and endoneurial inflammatory infiltrate is observed in all nerves, nerve roots, or plexus during segmental demyelination delivered by macrophages, especially in areas with lymphoid infiltrates [14]. The electrophysiological analysis shows that anti-GM1 antibodies occur more frequently in groups of patients with inexcitable axonal or nerve injury [7]. The AMAN study after $C$. jejuni infection provides strong evidence of the concept of molecular mimicry in the pathophysiology of immune neuropathy. The relationship between AMAN and previous Campylobacter infections fulfills four criteria that meet molecular mimicry: Epidemiological relationships between GBS and $C$. jejuni infections, identification of direct antibodies to host antigens (gangliosides) in patients, identification of microbial mimics on target antigens, and experimental neuropathy development in animal models [15].

Changes that occur due to GM1 immunization are seen both in the central nervous system and peripheral nerves. Although AMAN is peripheral neuropathy, we can also see slight changes in the lumbar bone marrow in this preparation. This study is expected to be the basis for making the first AMAN model in Indonesia. Provision of other adjunctive therapies can be given on this model, even better when compared with standard therapy.

\section{Conclusion}

The clinical picture of weakness with a trip monophasic and acute-GM1 immunized rabbits similar to that occurring in patients with GBS. GBS type of axonal has 
a dominant axonal involvement, characterized by similar Wallerian degeneration in HE staining, with only minimal demyelination occurs and infiltration of lymphocytes. Paralysis rabbits showed the same pathological changes predominantly axonal degeneration in peripheral nerves.

In conclusion, the inoculation experiment of rabbits with GM1 has been successfully proved in presenting a new model for molecular pathogenesis determination for axonal type. To see the better view of nerve regeneration in light microscopy, we suggest using another method such as Marchi. Hopefully, this model can be used in finding new alternative therapies for patients with GBS, more specifically in axonal types.

\section{Acknowledgments}

I would like to thank Sapta Prihartono, MD, surgeon, pediatric surgeon, for providing the facility to conduct this research.

\section{Authors' Contributions}

1. Ni Komang Sri Dewi Untari: Conceptualization, methodology, formal analysis, investigation, data curation, original draft preparation, review, and editing.

2. Kurnia Kusumastuti: Methodology, investigation, and review

3. M. Guritno Suryokusumo: Methodology, investigation, and review

4. I. Ketut Sudiana: Methodology, validation, and review

5. Tedy Juliandhy (submitting author): Methodology and editing

\section{References}

1. Tandel H, Pandya N, Pharmaceuticals A, Jani P. Guillainbarré syndrome (GBS): A review. Curr Med Res Opin.
2016;3(2):366-71.

2. Seneviratne U. Guillain-Barre syndrome. Postgrad Med J. 2000;76(902):774-82. https://doi.org/10.1136/ pgmj.76.902.774

PMid:11085768

3. Walling AD, Dickson G. Guillain-Barré Syndrome. 2013. p. 191-8.

4. Willison HJ. Peripheral neuropathies and anti-glycolipid antibodies. Brain 2002;125(12):2591-625. https://doi. org/10.1093/brain/awf272

PMid:12429589

5. Burns TM. Guillain-Barré syndrome. Semin Neurol. 2008;28(2):152-67.

PMid: 18351518

6. Thomas FP, Trojaborg W, Nagy C, Santoro M, Sadiq SA Latov $\mathrm{N}$, et al. Experimental autoimmune neuropathy with anti-GM1 antibodies and immunoglobulin deposits at the nodes of Ranvier. Acta Neuropathol. 1991;82(5):378-83. https://doi. org/10.1007/bf00296548 PMid:1767631

7. Yuki N, Yamada M, Koga M, Odaka M, Susuki K, Tagawa $Y$, et al. Animal model of axonal Guillain-Barré syndrome induced by sensitization with GM1 ganglioside. Ann Neurol. 2001;49(6):712-20. https://doi.org/10.1002/ana.1012 PMid:11409422

8. Willison $\mathrm{HJ}$, Jacobs BC, van Doorn PA. Guillain-Barré syndrome. Lancet. 2016;388(10045):717-27. https://doi.org/10.1016/ s0140-6736(16)00339-1

PMid:26948435

9. van Buskirk C. Clinical neurology. J Nerv Ment Dis. 1964;139(6):598

10. Nagai Y, Momoi T, Saito M, Mitsuzawa E, Ohtani S. Ganglioside syndrome, anewautoimmune neurologic disorder, experimentally induced with brain gangliosides. Neurosci Lett. 1976;2(2):107-11. https://doi.org/10.1016/0304-3940(76)90033-1

11. Carriel V, Garzón I, Alaminos M, Campos A. Evaluation of myelin sheath and collagen reorganization pattern in a model of peripheral nerve regeneration using an integrated histochemical approach. Histochem Cell Biol. 2011;136(6):709-17. https://doi. org/10.1007/s00418-011-0874-3 PMid:22038043

12. Abu Rafee M, Sharma GT. Short communication comparison of hematoxylin and eosin staining with and without pre treatment with marchi's solution on nerve samples for nerve degeneration and regeneration studies. Explor Anim Med Res. 2017;7(2):206.

13. Lichtman $\mathrm{H}$, Lichtman $\mathrm{H}$, Abbas A. Cellular and Molecular Immunology. Amsterdam, Netherlands: Elsevier; 2005.

14. Dalakas MC. Pathogenesis of immune-mediated neuropathies. Biochim Biophys Acta 2015;1852(4):658-66. PMid:24949885

15. Bourque PR, Chardon JW, Massie R. Autoimmune periphera neuropathies. Clin Chim Acta. 2015;449:37-42. https://doi. org/10.1016/j.cca.2015.02.039 